\title{
EL LUGAR Y EL IDIOMA EN EL ARBITRAJE
}

\author{
Mario Castillo Freyre \\ Rita Sabroso Minaya ${ }^{2}$ \\ Laura Castro Zapata ${ }^{3}$ \\ Jhoel Chipana Catalán ${ }^{4}$
}

\section{RESUMEN}

El artículo pretende aclarar lo relativo al lugar del arbitraje, así como los conceptos: circunstancias del caso y conveniencia de las partes, para la fijación del mismo a falta de acuerdo.

También se analiza el idioma del arbitraje y su determinación en ausencia de la voluntad de las partes.

\section{PALABRAS CLAVE}

- Arbitraje.

- Lugar del arbitraje.

- Idioma del arbitraje.

Como sabemos, el objetivo de un proceso arbitral es resolver una controversia sometida por las partes a la decisión de un tribunal arbitral. Sin embargo, para lograr tal fin, existen una serie de temas de vital importancia que van a permitir que el tribunal arbitral desarrolle las actuaciones arbitrales de manera adecuada y en concordancia con lo pactado por las partes $\mathrm{y}$, en su defecto, con lo establecido por la ley. En ese sentido, dos temas que resultan relevantes son los relacionados al lugar y al idioma en que se llevarán a cabo las actuaciones arbitrales. Teniendo ello en cuenta, a través del presente ensayo, vamos a analizar ambos conceptos.

\section{EL LUGAR DEL ARBITRAJE}

El lugar del arbitraje se encuentra regulado en el artícu- lo $35^{5}$ del Decreto Legislativo n. 1071 (en adelante, la Ley de Arbitraje), el mismo que establece lo siguiente:

Artículo 35.- Lugar del arbitraje

1. Las partes podrán determinar libremente el lugar del arbitraje. A falta de acuerdo, el tribunal arbitral determinará el lugar del arbitraje, atendiendo

Mario Castillo Freyre, Magíster y Doctor en Derecho, Abogado en ejercicio, socio del Estudio que lleva su nombre; profesor principal en la Pontificia Universidad Católica del Perú. Miembro de Número de la Academia Peruana de Derecho. Director de las colecciones Biblioteca de Arbitraje y Biblioteca de Derecho de su Estudio. Decano de la Facultad de Derecho de la Universidad Católica San Pablo de Arequipa. www.castillofreyre.com.

2 Rita Sabroso Minaya, Abogada del Estudio Mario Castillo Freyre. Ha sido Profesora de Arbitrajes Especiales en la Facultad de Derecho de la Universidad de Lima. Con estudios en la Maestría de Derecho de la Competencia y Propiedad Intelectual en la Pontificia Universidad Católica del Perú.

3 Laura Castro Zapata, Abogada por la Universidad Femenina del Sagrado Corazón. Magíster en Derecho, con mención en Derecho Empresarial, por la Universidad de Lima. Ha concluido sus estudios en el Doctorado en Derecho de la Pontificia Universidad Católica del Perú. Ejerce la profesión en Laura Castro Zapata Abogada Arbitrajes. También se desempeña como árbitro.

4 Jhoel Chipana Catalán, Abogado por la Pontificia Universidad Católica del Perú, con estudios concluidos de Maestría en la Escuela de Posgrado de la Universidad Nacional Mayor de San Marcos. Profesor en la Universidad de San Martín de Porres y Abogado en el Estudio Mario Castillo Freyre.

5 La Ley Modelo Uncitral establece sobre este tema lo siguiente:

Artículo 20.- Lugar del arbitraje

1) Las partes podrán determinar libremente el lugar del arbitraje. En caso de no haber acuerdo al respecto, el tribunal arbitral determinará el lugar del arbitraje, atendidas las circunstancias del caso, inclusive las conveniencias de las partes. 
a las circunstancias del caso y la conveniencia de las partes.

2. Sin perjuicio de lo dispuesto en el numeral anterior, el tribunal arbitral podrá, previa consulta a las partes, reunirse en cualquier lugar que estime apropiado para oír a los testigos, a los peritos o a las partes, o para examinar o reconocer objetos, documentos o personas. El tribunal arbitral podrá llevar a cabo deliberaciones en cualquier lugar que estime apropiado.

Antes de ingresar al análisis del contenido del artículo 35 del Decreto Legislativo $\mathrm{n}$. 1071, debemos resaltar que nos encontramos ante una norma de carácter dispositivo.

El artículo 35, inciso 1 de la Ley de Arbitraje, otorga libertad a las partes para determinar libremente el lugar en que se desarrollará el arbitraje. No debemos olvidar que los límites más importantes a este libre ejercicio de la autonomía, son el respeto de los derechos de igualdad de partes, autonomía y contradicción.

Ahora bien, se debe aclarar en el primer inciso del artículo bajo análisis, cuáles son los alcances de dos frases, a saber: «circunstancias del caso» y «conveniencia de las partes».

Así, Tovar Gil ${ }^{6}$ señala que por «circunstancias del caso» debe entenderse que los árbitros deben tomar en consideración las características particulares de la disputa. No existe un recetario determinado que establezca qué características priman a la hora de la elección, por lo que ésta deberá realizarse caso por caso. Por ejemplo, podrá escogerse el lugar en donde se encuentre la mayor cantidad de pruebas, o el lugar donde domicilie la mayoría de los árbitros, o el lugar neutral más próximo a las partes y árbitros; entre otros.

De la misma forma, continúa la citada profesora, existe debate sobre el concepto «conveniencia de las partes», en tanto se trata de un concepto subjetivo de difícil definición.
Lo que queda claro es que el árbitro no puede simplemente designar un lugar a su mero arbitrio, sino que deberá considerar los criterios que a su juicio determinen lo que se ha venido en llamar la conveniencia de las partes, a fin de asegurar que el arbitraje sea lo más justo y eficiente posible.

Por nuestra parte, creemos que ambos conceptos deben tomarse en cuenta, ya que uno deberá reflejar, necesariamente, al otro; ello, en el sentido de que no creemos que sea coherente elegir un lugar para el arbitraje que atienda a las circunstancias del caso en particular, que colisione con los intereses de las partes. Asimismo, sobre el primer concepto, los árbitros deberán tener presentes las circunstancias en que se produjo el conflicto de intereses, $y$, de otro lado, para atender a los intereses de las partes, podrían consultarles o solicitarles su opinión al respecto, de manera que con toda esa información se pueda tomar la mejor decisión.

2) Sin perjuicio de lo dispuesto en el párrafo precedente, el tribunal arbitral podrá, salvo acuerdo en contrario de las partes, reunirse en cualquier lugar que estime apropiado para celebrar deliberaciones entre sus miembros, para oír a los testigos, a los peritos o a las partes, o para examinar mercancías u otros bienes o documentos.

De la misma forma, la Ley de Arbitraje española del año 2003, sobre este particular estipula lo siguiente:

Artículo 26.- Lugar del arbitraje

1. Las partes podrán determinar libremente el lugar del arbitraje. A falta de acuerdo, lo determinarán los árbitros, atendidas las circunstancias del caso y la conveniencia de las partes.

2. Sin perjuicio de lo dispuesto en el apartado anterior, los árbitros podrán, previa consulta a las partes y salvo acuerdo en contrario de éstas, reunirse en cualquier lugar que estimen apropiado para oír a los testigos, a los peritos o a las partes, o para examinar o reconocer objetos, documentos o personas. Los árbitros podrán celebrar deliberaciones en cualquier lugar que estimen apropiado.

Dentro de la normativa nacional, el artículo 35 del Decreto Legislativo n. ${ }^{\circ} 1071$, bajo estudio, tiene como antecedente inmediato el numeral 109 de la derogada Ley General de Arbitraje del año 1996, Ley n. ${ }^{\circ} 26572$. Así, dicho precepto señalaba:

Artículo 109.- Lugar del arbitraje

Las partes podrán determinar libremente el lugar del arbitraje. En caso de no haber acuerdo al respecto, el tribunal arbitral determinará el lugar del arbitraje atendiendo a las circunstancias del caso, inclusive las conveniencias de las partes. Sin perjuicio de lo dispuesto en el párrafo precedente, el tribunal arbitral podrá, salvo acuerdo en contrario de las partes, reunirse en cualquier lugar que estime apropiado para celebrar deliberaciones entre sus miembros, para oír a los testigos, a los peritos o a las partes, o para examinar mercancías u otros bienes o documentos.

6 TOVAR GIL, María del Carmen. «Lugar del arbitraje». En AA.VV. Comentarios a la ley peruana de arbitraje. Lima: IPA, 2011, tomo I, p. 398. 
Sobre este mismo punto, Yañez Velasco ${ }^{7}$ sostiene que como sea que los árbitros han de tener en cuenta las circunstancias del caso, deberán conocer su contenido para evaluar, y no cabe pensar que puedan determinar correctamente el lugar del arbitraje antes de apreciar el objeto del arbitraje. Éste se determina en la demanda y en la contestación, y cuando las partes no prefijaron el lugar de arbitraje en el convenio arbitral o con alegaciones previas a esos dos escritos y lo pretendan hacer después, al tiempo o a continuación de sus alegaciones iniciales (demanda y contestación), no parece oportuno delimitar el lugar por los árbitros sino hasta conocer un mínimo contenido de la controversia, vale decir, sus circunstancias. Evidentemente, las actuaciones arbitrales deberán ir desarrollándose en algún lugar. Para ello sería posible la determinación de un lugar provisional 0 , simplemente, la práctica de la presentación de la demanda y la contestación con cualquier sitio donde se encuentre el árbitro, dato luego no vinculante sobre la determinación del lugar del arbitraje ni vía tácita para su especificación.

Ahora bien, pese a estos matices, generalmente, las partes van a acordar el lugar del arbitraje aunque, de manera usual, en los convenios no se señale la ciudad en donde éste se va a realizar.

En la mayoría de ocasiones ello se sobreentiende, en la medida de que normalmente ambas partes radican o tienen sus domicilios en la misma ciudad, y, no solo eso, sino que los árbitros también residen en la misma ciudad.

Por lo general, en los arbitrajes nacionales, donde no se indique el lugar del arbitraje, nos encontraremos en presencia de situaciones en las cuales el arbitraje se desarrollará en Lima, por obvias razones. Creemos que ese tema no constituye objeto de discusión o debate; simplemente, se producen situaciones naturales en este sentido que son, por lo demás, las más comunes.

Ahora, si bien existe una norma en el sentido de que, a falta de acuerdo, el tribunal arbitral determinará el lugar del arbitraje atendiendo a las circunstancias del caso y la conveniencia de las partes, lo que ocurre en la práctica arbitral, en muchos casos, es que las circunstancias aconsejan que el arbitraje se desarrolle en la ciudad de Lima, porque generalmente todos los actores, incluidos los miembros del tribunal, radican en dicha ciudad.

Es verdad que existen algunas situaciones en las cuales el tema lleva a duda, como las referidas al hecho de que no se establece el lugar del arbitraje en el convenio arbitral. Se trata, pues, de convenios arbitrales para tribunales ad-hoc, en donde una parte radica en una determinada localidad, otra en una localidad distinta y los árbitros son de esta última localidad.
Concretamente, a título ejemplificativo, esto se presenta cuando una de las partes es una entidad estatal de provincia y los árbitros y la empresa son de Lima. Ahí sería evidente que el tribunal arbitral debería tener su sede en Lima.

Cuando ambas partes son de una localidad distinta y los integrantes del tribunal son de Lima, es donde empiezan los problemas. El tribunal, generalmente, establecerá como lugar del arbitraje la ciudad de Lima, a menos que las partes quieran asumir los costos de su traslado. Ahí, lo usual será que el tribunal sopese las posibilidades de llevar a cabo el arbitraje en su lugar natural de residencia o de conducirlo en la localidad en que radican las partes.

Esto también depende de la disponibilidad de tiempo del tribunal y no sólo de los recursos de las partes; de tal manera que en situaciones como éstas, finalmente, es el tribunal el que va a decidir, pero siempre deberá atender a la voluntad de las partes y a las posibilidades económicas de sufragar los traslados y costos adicionales.

Generalmente, en estos temas los tribunales arbitrales buscan lo que resulte más económico y conveniente a las partes.

Ahora bien, respecto al inciso 1 del artículo 35, bajo comentario, resulta importante citar un problema identificado por Monserrat de Hoyos, ${ }^{8}$ quien se pregunta: ¿qué pasará cuando

YÁÑEZ VELASCO, Ricardo. Comentarios a la nueva Ley de Arbitraje. Valencia: Tirant lo Blanch, 2004, p. 507.

8 DE HOYOS SANCHO, Monserrat. «Lugar del arbitraje». En Comentarios prácticos a la Ley de Arbitraje. Valladolid: Editorial Lex Nova, 2004, p. 483. 
los árbitros ya comunicaron su designación a las partes y éstas, luego, pretenden imponer al tribunal arbitral una sede distinta de la que en principio aparecía designada, bien en el convenio, bien en el reglamento o bien en el propio acuerdo que las partes han tomado?

Creemos, junto a De Hoyos, que el acuerdo de las partes sobre el lugar del arbitraje deberá imponerse solo en la medida en que los árbitros conozcan esa circunstancia con anterioridad, bien por contenerse ya en el convenio arbitral o bien porque se pacta después de la firma del convenio, pero se da a conocer a los árbitros antes de que éstos decidan si aceptan o rechazan el encargo que se les propone. De no suscribirse esta interpretación, podría darse la circunstancia de que los árbitros se vieran sometidos a la concreción de un extremo del procedimiento por parte de los litigantes, de gran trascendencia para los integrantes del tribunal arbitral, que fuera in fine en contra de sus propios intereses profesionales o personales.

Sin lugar a dudas, y sin perjuicio de lo señalado, en este supuesto podría recurrirse a la figura de la remoción, en el sentido de que los árbitros no podrán, por cuestiones de hecho, seguir desenvolviéndose como tales.

Por otro lado, el inciso 2 del artículo 35 de la Ley de Arbitraje, establece que sin perjuicio de lo dispuesto en el numeral anterior, el tribunal arbitral podrá, previa consulta a las partes, reunirse en cualquier lugar que estime apropiado para oír a los testigos, a los peritos o a las partes, 0 para examinar o reconocer objetos, documentos o personas.

Es natural que la labor del tribunal no pueda y no deba estar circunscrita a lo que significa la sede del tribunal arbitral, porque hay determinadas diligencias que no se van a poder realizar, precisamente, en esa sede.

En concreto, podríamos hablar de la posibilidad de entrevistar a determinados testigos que no se encuentren en la sede del tribunal y que su traslado resulte imposible por razones de salud o que, incluso, encontrándose en la misma ciudad no puedan movilizarse. También se podría pensar en inspecciones arbitrales que se realicen fuera de la sede del tribunal arbitral, ya sea dentro de la misma ciudad o fuera de ella, y otros casos de similar naturaleza. En esas circunstancias el tribunal arbitral podrá disponer la realización de actuaciones fuera de la sede.

En adición a ello, hay que entender que el tribunal podría incluso dentro de la misma ciudad variar el lugar físico donde se realicen las actuaciones, sin que se varíe finalmente la sede. Esto no implica, de modo alguno, una variación o cambio de la sede. La sede sigue siendo la misma, a pesar de que transitoriamente se realicen actuaciones en otros lugares. Un caso típico es aquél en el que algunas actuaciones tienen que realizarse fuera de la sede del tribunal por motivos de espacio, en razón del número elevado de litis consortes, o incluso, de personas que integren o aseso- ren a una sola parte, que no quepan dentro de la sede del tribunal arbitral.

El citado inciso 2 del artículo 35 de la Ley de Arbitraje, señala, adicionalmente, que el tribunal arbitral podrá llevar a cabo deliberaciones en cualquier lugar que estime apropiado.

Esto es de gran importancia porque, en realidad, la sede es un lugar meramente formal, que no debe atar las consideraciones en torno al trabajo del tribunal, sino simplemente se conviene para cuestiones de orden formal, para los escritos que serán presentados por las partes en la sede. Oficialmente, las audiencias, salvo disposición distinta del tribunal, serán realizadas en la sede. Sin embargo, el tribunal no siempre se deberá reunir allí.

Es más, el tema relativo a las deliberaciones es importante porque, generalmente, la sede implica consideraciones de formalidad, en el sentido de que el tribunal deba desplazarse oficialmente a un determinado lugar, como es el caso de un Estudio de Abogados, un Centro de Arbitraje, etc.

Sin embargo, los árbitros -con toda facilidad- podrán reunirse en el lugar que consideren conveniente: otro lugar, otro Estudio de Abogados, la casa de uno de los árbitros, un restaurante, etc., para poder deliberar. Esto brinda más facilidades al tribunal arbitral para poder reunirse. Incluso, podríamos pensar en una especie de sede virtual, concepto no introducido en la Ley de Arbitraje, que es perfectamente 
pasible de ser asimilado a estas normas.

La sede virtual se presenta cuando los miembros del tribunal arbitral deliberan empleando el Internet. Entonces, allí uno se podría preguntar sobre cuál es la sede de la deliberación, pues, en realidad, no hay una sede; no hay un lugar físico, más allá del espacio material desde donde cada uno de ellos esté conectado a un ordenador y desde donde se encuentre enviando estos mensajes.

Además, podríamos preguntarnos si en verdad es importante que el concepto de la sede del tribunal arbitral se mantenga como un concepto absolutamente unívoco y en términos físicos, como tradicionalmente se ha entendido.

Pensamos que la sede del tribunal arbitral debe servir sólo para cuestiones formales. La regla debe ser que en tanto y en cuanto, la sede del tribunal arbitral perturbe el adecuado funcionamiento del tribunal en relación a los árbitros entre sí, o de los árbitros con las partes, debe primar la flexibilidad, naturalmente, siempre y cuando esta flexibilidad no perjudique el derecho de defensa de las partes.

En otras palabras, el tribunal arbitral no podría cometer la arbitrariedad de plantear la realización de una audiencia en un lugar extremadamente alejado, en donde por razones económicas sólo pueda asistir una de las partes en perjuicio de la otra, variando de facto, la realización de estos actos en la sede del tribunal arbitral.
Entonces, además es necesario señalar que este tipo de situaciones usualmente no ofrecen mayores problemas porque las audiencias se realizan, por lo general, en la sede del tribunal arbitral, salvo cuestiones excepcionales. O sea, tampoco es que el tribunal arbitral disponga una serie indefinida de actos procesales fuera de la sede.

Cuando se recurre a lugares fuera de la sede ello obedece, sin duda, a razones de excepción, que motivan un proceder de esta naturaleza.

Resulta necesario señalar que la sede del tribunal arbitral debe proporcionar a las partes y al propio tribunal las condiciones de seguridad, eficiencia e idoneidad, propias para el desarrollo del proceso arbitral.

No nos cabe duda de que si la sede se constituyera en un Estudio de Abogados donde hubiese un reloj automático, una secretaria permanente que reciba dentro del horario establecido los escritos, los examine, los selle, atienda a las personas que van a venir a las audiencias, donde haya directorios, equipos de cómputo, implementos para desarrollar las audiencias, etc., sin duda, será una sede idónea para un tribunal arbitral.

En los tribunales, generalmente, debería tenderse a que este tipo de instalaciones sean las que primen como sede, a efectos de que el desarrollo de las audiencias no revista problemas logísticos.

Además, hay algo muy importante, y es que la confianza de las partes no debe perderse, de tal manera que esa seriedad que brinden estos mecanismos de recepción de documentos, de atención a innumerables llamadas telefónicas, correos electrónicos, etc., va a fomentar la confianza de las partes en el desarrollo del proceso.

Ahora bien, uno de los supuestos no tratados por la Ley es el relativo al cambio de sede del tribunal arbitral.

Al fin y al cabo, si bien es cierto que la Ley expone en el artículo 35 que son los tribunales arbitrales los que van a disponer -en última instancia- dónde será la sede del tribunal arbitral, esta situación -en la medida en que se fija en el acta de instalación del tribunal arbitral- constituye parte de ese contrato que celebran las partes y los árbitros en el acta referida.

En este sentido, lo que habría que considerar es si el tribunal arbitral puede variar la sede sin contar con el asentimiento de las partes.

Sobre este particular, se presentan situaciones muy comunes. Por ejemplo, el tribunal arbitral designa como sede, lo que es muy usual en los arbitrajes ad-hoc, al Estudio de Abogados del Presidente o de alguno de los miembros del tribunal, específicamente de aquél que tenga una oficina con mayores condiciones de idoneidad para que ella sea sede del tribunal.

En situaciones como éstas, podría ocurrir que el abogado cuyo Estudio se designa como sede, deje de ser parte de ese Estudio o, simplemente, mude sus oficinas a otro lugar. 
Entonces, es evidente que el inmueble cuya dirección sería anotada como sede del tribunal arbitral, ya no va a poder seguir atendiendo los requerimientos del tribunal, porque no guarda relación alguna con los árbitros.

Así, en situaciones como éstas lo natural e imperativo será un cambio de sede, debido a las circunstancias bajo las que nos encontramos.

Es evidente que ese cambio de sede va a ser dispuesto por el propio tribunal arbitral.

Por lo general, un cambio de sede, más allá de ser excepcional, no debe ameritar ninguna observación por las partes; ello, naturalmente, en tanto y en cuanto esa nueva sede revista condiciones similares o incluso mejores que la anterior.

Una observación como ésta debería ser declarada fundada por el tribunal arbitral y podría merecer observación, en la medida de que la nueva sede incluya elementos de degradación en cuanto a las seguridades para el desarrollo del proceso. Por ejemplo, que se traslade al domicilio de uno de los miembros del tribunal arbitral, en donde no haya reloj automático para la recepción de documentos, donde no haya secretaria que los reciba, o situaciones que verdaderamente hagan que no haya las mínimas condiciones de seguridad para las partes. Incluso, también podría objetarse la modificación, en la medida en que se trate de un lugar absolutamente alejado, o ubicado en una zona muy peligrosa de la ciudad, cuyo acceso sea difícil, etc. Entonces, las partes sí podrían formular alguna observación para que el tribunal reconsidere esa decisión y establezca una sede que revista las condiciones de idoneidad que tenía la anterior.

Todo cambio de sede, sin duda, puede ser dispuesto por el tri- bunal arbitral dentro de estos parámetros y consideramos que, en efecto, no viola -en lo absoluto- el debido proceso, ya que -más bien- el proceder se encuentra derivado de cuestiones imperativas o de conveniencia del propio tribunal que lo lleven a actuar de esta manera.

Naturalmente, si ese cambio de sede revistiera las objeciones que acabamos de anotar, las partes podrían impugnarlo, estimando que afecta los parámetros establecidos en el acta de instalación, las condiciones de la sede original, y que, por último, no brinda las garantías necesarias para el respeto al debido proceso arbitral.

\section{EL IDIOMA DEL ARBITRAJE}

El idioma en el que se desarrollarán las actuaciones arbitrales, se encuentra regulado en el artículo $36^{9}$ de la Ley de Arbitraje, que a la letra establece lo siguiente:

9 La Ley Modelo Uncitral establece sobre este tema lo siguiente:

Artículo 22.- Idioma

1) Las partes podrán acordar libremente el idioma o los idiomas que hayan de utilizarse en las actuaciones arbitrales. A falta de tal acuerdo, el tribunal arbitral determinará el idioma o los idiomas que hayan de emplearse en las actuaciones.

Este acuerdo o esta determinación será aplicable, salvo que en ellos mismos se haya especificado otra cosa, a todos los escritos de las partes, a todas las audiencias, y a cualquier laudo, decisión o comunicación de otra índole que emita el tribunal arbitral.

2) El tribunal arbitral podrá ordenar que cualquier prueba documental vaya acompañada de una traducción al idioma o los idiomas convenidos por las partes o determinados por el tribunal arbitral.

De la misma forma, la Ley de Arbitraje española del año 2003, sobre este particular estipula:

Artículo 28.- Idioma del arbitraje

1. Las partes podrán acordar libremente el idioma o los idiomas del arbitraje. A falta de acuerdo, y cuando de las circunstancias del caso no permitan delimitar la cuestión, el arbitraje se tramitará en cualquiera de las lenguas oficiales en el lugar donde se desarrollen las actuaciones. La parte que alegue desconocimiento del idioma tendrá derecho a audiencia, contradicción y defensa en la lengua que utilice, sin que esta alegación pueda suponer la paralización del proceso.

Salvo que en el acuerdo de las partes se haya previsto otra cosa, el idioma o los idiomas establecidos se utilizarán en los escritos de las partes, en las audiencias, en los laudos y en las decisiones o comunicaciones de los árbitros, sin perjuicio de lo señalado en el párrafo primero.

En todo caso, los testigos, peritos y terceras personas que intervengan en el procedimiento arbitral, tanto en actuaciones orales como escritas, podrán utilizar su lengua propia. En las actuaciones orales se podrá habilitar como intérprete a cualquier persona conocedora de la lengua empleada, previo juramento o promesa de aquélla.

2. Los árbitros, salvo oposición de alguna de las partes, podrán ordenar que, sin necesidad de proceder a su traducción, cualquier documento sea aportado o cualquier actuación realizada en idioma distinto al del arbitraje. 
Artículo 36.- Idioma del arbitraje

1. Las partes podrán acordar libremente el idioma o los idiomas que hayan de utilizarse en las actuaciones arbitrales. A falta de acuerdo, el tribunal arbitral determinará el idioma o los idiomas del arbitraje, atendidas las circunstancias del caso. Salvo que en el acuerdo de las partes o en la decisión del tribunal arbitral se haya previsto algo distinto, el idioma o los idiomas establecidos se utilizarán en los escritos de las partes, en las audiencias, en los laudos y en las decisiones o comunicaciones del tribunal arbitral.

2. El tribunal arbitral podrá ordenar que, sin necesidad de proceder a su traducción, cualquier documento sea aportado o cualquier actuación sea realizada en idioma distinto al del arbitraje, salvo oposición de alguna de las partes.
Esta norma tiene carácter meramente dispositivo, porque señala que las partes podrán acordar libremente el idioma o los idiomas que haya que utilizar en las actuaciones arbitrales.

Por lo general, no existe problema alguno en materia de arbitraje nacional, porque ambas partes utilizan o emplean el mismo idioma (el español). Tovar $\mathrm{Gil}^{10}$ sostiene que a lo largo de los años la cuestión del idioma del arbitraje ha pasado más o menos inadvertida en la doctrina nacional. Con la globalización del sistema económico internacional y la política sostenida de inserción comercial del Perú en los mercados del mundo, se multiplica la posibilidad de enfrentar disputas con socios comerciales de los rincones más alejados del planeta. La idea de enfrentar un arbitraje con partes que no compartan el mismo idioma, no es ya para nada distante y pasa a convertirse en un tema que debe estar presente al momento de la redacción de la cláusula arbitral y esto es algo que los abogados debemos tener en cuenta si hemos de especializarnos en arbitraje.

Los problemas en materia de idioma del arbitraje se presentan, normalmente, en los arbitrajes internacionales, cuando las dos partes no tienen un idioma natural en común. La pluralidad de idiomas puede presentarse y vincularse a las mismas partes interesadas (que cada una tenga un idioma diferente) o a lugares predeterminados para actuaciones concretas (tomar testimonios en el extranjero, por ejemplo) ${ }^{11}$.

En esos casos, podría producirse un acuerdo para llevar el proceso arbitral en un idioma distinto al de ambas. Por ejemplo, una parte es una empresa francesa y la otra es una empresa española. Aquí, las partes podrán acordar que el idioma del arbitraje sea el inglés. No habría ningún problema para tal efecto.

(Este artículo está redactado conforme a la Ley $n .^{\circ} 11 / 2011$, de 20 de mayo de 2011, a través de la cual se reforma la Ley $n .{ }^{\circ}$ 60/2003 de Arbitraje y de regulación del arbitraje institucional en la Administración General del Estado, de 23 de diciembre).

Dentro de la normativa nacional, el artículo 36 del Decreto Legislativo $n .^{\circ} 1071$, bajo estudio, tiene como antecedente inmediato el artículo 111 de la derogada Ley de Arbitraje del año 1996, Ley n. ${ }^{\circ} 26572$. Así, dicho precepto señalaba lo siguiente:

Artículo 111.- Idioma

Las partes podrán acordar libremente el idioma o los idiomas que hayan de utilizarse en las actuaciones arbitrales. A falta de tal acuerdo, el tribunal arbitral determinará el idioma o los idiomas que hayan de emplearse en las actuaciones. Este acuerdo o esta determinación será aplicable, salvo que en ellos mismos se haya especificado otra cosa, a todos los escritos de las partes, a todas las audiencias, y a cualquier laudo, decisión o comunicación de otra índole que emita el tribunal arbitral. El tribunal arbitral podrá ordenar que cualquier documento esté acompañado de una traducción al idioma o los idiomas convenidos por las partes o determinados por el tribunal arbitral.

Finalmente, el artículo que nos corresponde analizar también posee antecedente en la Ley de Arbitraje peruana del año 1992, Decreto Ley.$^{\circ}$ 25935. Se trata del numeral 97, el cual señalaba:

Artículo 97. - Las partes podrán acordar libremente el idioma o los idiomas que hayan de utilizarse en las actuaciones arbitrales. A falta de tal acuerdo, el tribunal arbitral determinará el idioma o los idiomas que hayan de emplearse en las actuaciones. Este acuerdo o esta determinación será aplicable, salvo que en ellos mismos se haya especificado otra cosa, a todos los escritos de las partes, a todas las audiencias, y a cualquier laudo, decisión o comunicación de otra índole que emita el tribunal arbitral.

El tribunal arbitral podrá ordenar que cualquier documento esté acompañado de una traducción al idioma o a los idiomas convenidos por las partes o determinados por el tribunal arbitral.

10 TOVAR GIL, María del Carmen. «Idioma del arbitraje». En AA.VV. Comentarios a la ley peruana de arbitraje. Op. cit., tomo I, p. 407.

11 YÁÑEZ VELASCO, Ricardo. Op. cit., p. 530. 
Ahora, otro supuesto de hecho será aquél en el cual existan discrepancias sobre ese aspecto.

Allí rige la norma del artículo 36 de la Ley de Arbitraje, cuando señala que a falta de acuerdo, si fuese un tribunal que se rigiese por la ley peruana, el tribunal determinará el idioma o los idiomas del arbitraje, atendiendo a las circunstancias del caso.

Ello, porque el proceso podría llevarse, tanto en un idioma como en varios idiomas.

El tribunal podría aceptar que el proceso se lleve en dos idiomas con documentos traducidos, si fuese el caso, al idioma de la parte contraria. O que los escritos se traduzcan al idioma de la parte contraria, o que las audiencias se realicen -alternativamente- en los idiomas fuente con traducción simultánea; ello, para el desarrollo más expeditivo de las actuaciones arbitrales.

No nos cabe duda de que todas estas consideraciones adicionales podrían encarecer los costos del arbitraje, pero si ello facilita el desarrollo del mismo y hace que las partes se encuentren conformes con el proceso, pues no habrá problema alguno para que el tribunal proceda de esa forma.

Incluso podríamos hablar de la posibilidad de que un proceso se lleve en más de dos idiomas, en la medida de que no solo estemos en presencia de partes que tengan idiomas diferentes, sino de árbitros que puedan tener idiomas diferentes y que quieran emplear sus idiomas naturales para el desarrollo de las audiencias, contando con traducción simultánea en todas las actuaciones en las que intervengan.

Además, no hay que olvidar que los idiomas son el lenguaje de comunicación de las personas y tampoco hay que hacerse muchos problemas en torno al idioma de un proceso, en la medida en que las facilidades que hoy brindan los medios tecnológicos de traducción simultánea de documentos escritos o los traductores, las carreras de traducción e interpretación, las posibilidades de contar con asistentes en esta materia, etc., delinean todo un marco lo suficientemente amplio como para hacer que, independientemente del idioma oficial de ese arbitraje, las partes entiendan perfectamente qué es lo que su parte contraria está expresando en las audiencias o en los escritos, e incluso con respecto a las intervenciones del propio tribunal y de terceros, como podría ser el caso de peritos, de testigos o de personas que sean parte de lo que el tribunal arbitral observe en una inspección arbitral, por citar un ejemplo.

El artículo 36, inciso 1 de la Ley de Arbitraje del Perú, agrega que salvo que en el acuerdo de las partes o en la decisión del tribunal arbitral se haya previsto algo distinto, el idioma o los idiomas establecidos se utilizarán en los escritos de las partes, en las audiencias, en los laudos y en las decisiones o comunicaciones del tribunal arbitral.
Esto implica, evidentemente, la necesidad de que todo el proceso se siga en el «idioma oficial» que ese proceso hubiere establecido.

Ahora, con todo lo que ya hemos señalado, el inciso 2 del artículo 36 anota que el tribunal arbitral podrá ordenar que, sin necesidad de proceder a su traducción, cualquier documento sea aportado o cualquier actuación sea realizada en idioma distinto al del arbitraje, salvo oposición de alguna de las partes.

Naturalmente, esto brinda facilidad a las partes para poder aportar documentos, en los idiomas originales que no sean propios del idioma oficial adoptado por el tribunal.

Sin embargo, creemos que en estos casos, si se tratara de documentos en idioma distinto al oficial, la parte deberá, por una cuestión de práctica usual, adjuntar también su traducción. Esta traducción no necesariamente tiene que ser oficial, salvo que sea objetada por la parte contraria, caso en el cual entendemos que el tribunal debería declarar fundada esa oposición, en la medida en que debe tenerse certeza y pleno entendimiento de aquello que se está llevando adelante en el tribunal y es que no debemos olvidar que escoger un idioma oficial tiene su razón de ser en ofrecer la seguridad a las partes y a quienes intervienen en el arbitraje.

Tovar Gil ${ }^{12}$ reconoce un problema que en la práctica po-

12 TOVAR GIL, María del Carmen. «Idioma del arbitraje». En AA.VV. Comentarios a la ley peruana de arbitraje. Op. cit., tomo I, p. 409. 
dría presentarse. Señala que existe un inconveniente relacionado con qué idioma debe usarse en las comunicaciones entre las partes; entre ellas y la institución arbitral y entre las partes y los árbitros, desde el momento en que se solicita el arbitraje, hasta el momento en que los árbitros determinan el idioma aplicable al procedimiento.

En efecto, continúa la citada profesora, si no hay un idioma establecido y ambas partes son de diversos países, con distintos idiomas, puede surgir el problema relativo a cuál es el idioma que debe regir sus comunicaciones. Debemos sin embargo partir de que, por lo general, la cláusula arbitral forma parte de un contrato y si la situación es la descrita, es decir, si las partes del contrato no comparten un idioma, es muy probable que el contrato, aun cuando no haya definido el idioma del arbitraje, sí haya establecido un idioma para las comunicaciones entre las partes. En todo caso, de no ser así, el contrato estará redactado en un idioma y debería entenderse que las comunicaciones se harán en el idioma en que esté redactado el contrato. Puede sin embargo, darse el caso, que es relativamente frecuente, en que el contrato se haya redactado en dos idiomas. En tal supuesto, deberíamos entender que, para estar seguros del valor de las comunicaciones que se envía a la otra parte, ellas deben hacerse en los dos idiomas, o por lo menos en el idioma que coincide con el de la otra parte, corriendo las traducciones por cuenta de quien dirige la comunicación.

En todo caso, creemos que no debería asumirse que no cabe comunicarse en un idioma distinto de aquél o aquéllos en que se ha redactado el contrato. 